\title{
The Kosztin-Leggett Nonlocal Effect on the Magnetic Penetration Depth in Superconductors with Nodes in the Gap
}

\author{
Ismardo Bonalde \\ Centro de Física, Instituto Venezolano de Investigaciones Científicas, Apartado 21874, Caracas 1020-A, Venezuela
}

Received on 1st July, 2003.

\begin{abstract}
The effect of nonlocal electrodynamics is considered in finite samples. It is found for such samples that nonlocality modifies not only the in-plane penetration depth $\lambda_{\|}$, as predicted by Kosztin and Leggett, but also the out-of-plane penetration depth $\lambda_{\perp}$. The $\mathrm{Sr}_{2} \mathrm{RuO}_{4}$ data are reinterpreted taking into account the contribution from $\lambda_{\perp}$.
\end{abstract}

\section{Introduction}

Penetration depth measurements have played a very fundamental role in the study of the gap symmetry of all unconventional superconductors. For instance, from magnetic penetration depth measurements it was first suggested the $d$ wave symmetry for the energy gap in high critical temperature superconductors (HTSC) [1]. It is expected that, for superconducting energy gaps with nodes on the Fermi surface, the deviation of the magnetic penetration depth $\lambda(T)$ from its zero temperature value goes as a power law in temperature, $\Delta \lambda(T)=\lambda(T)-\lambda(0) \propto T^{\alpha}$, at temperatures well below the critical temperature $T_{c}$. The exponent $\alpha$ depends on gap geometry, dimensionality and the rate at which the gap vanishes near the zeroes. For line nodes in three dimensional systems, like those with a $d$-wave symmetry, $\Delta \lambda(T) \propto T$ (the same conclusion holds for point nodes in two dimensional systems). In these systems, however, the penetration depth has been found in some cases to cross over from linear to quadratic temperature dependence at a temperature $T^{*}<<T_{c}$. Such a crossover has been explained theoretically as due to impurities [2] or nonlocal electrodynamics [3].

The impurity model considers that a dilute concentration of strong scatterers in the unitary limit in unconventional superconductors with line nodes causes a finite residual density of states near the nodes and negligible suppression of $T_{c}$. Thus, as $T \rightarrow 0$ a "gapless" temperature behavior [4] $\Delta \lambda(T) \propto T^{2}$ should be expected for such superconductors, and the crossover in $\Delta \lambda(T)$ is predicted to occur at $T^{*} \sim \sqrt{\Gamma \Delta_{0}}$. Here, $\Gamma$ is the scattering rate and $\Delta_{0}$ is the energy gap maximum. The nonlocal model supposes that the local condition $\lambda(0) / \xi_{0}>>1$, for which the spatial variation of the electromagnetic field over the extent of the Cooper pair is neglected and the pair is taken as a point object, is not satisfied at temperatures well below $T_{c}$ in most unconventional superconductors. Here $\xi_{0} \equiv \hbar \nu_{F} / \pi \Delta_{0}$ is the BCS coherence length. The nonlocal model is based on the fact that in superconductors with an anisotropic energy gap with nodes on the Fermi surface the coherence length $\xi_{0}(k)=\hbar \nu_{F} / \pi \Delta(k)$. Kosztin and Leggett (KL) [3] suggested for the local condition

$$
\frac{\lambda(0)}{\xi_{0}(k)}=\frac{\lambda(0)}{\xi_{0}} \frac{|\Delta(k)|}{\Delta_{0}}>>1,
$$

Thus near the nodes, where $\Delta(k) \rightarrow 0$, the local condition would no longer hold, and the superconductor would become nonlocal. KL predicted for very clean, semi-infinite superconductors with line nodes that nonlocal electrodynamics would affect the magnetic penetration depth only when the exciting field is applied perpendicular to the $a b$ plane; that is to say, only $\lambda_{\|}(T)$ would be perturbed by nonlocality. They proposed that $\Delta \lambda_{\|}(T) \propto T^{2}$ below a $T_{N L}^{*}=\Delta_{0} \xi_{0} / \lambda(0)<<T_{c}$.

The impurity effect has been confirmed in $\mathrm{Zn}$-doped YBCO [5] and has been used to explain data from organic superconductors [6] and various HTSC [7]. On the other hand, the KL nonlocal effect has been difficult to prove, and only recently some evidence for its existence has been obtained. The importance of testing experimentally the validity of the KL effect is because it would provide a mechanism for the observed crossover from linear to quadratic temperature dependence of the magnetic penetration depth in very clean (impurity effect unapplicable) superconductors with nodes in the energy gap. Here the KL nonlocal effect is discussed in finite superconductors with line nodes. It is found that the out-of-plane component of the penetration depth, $\lambda_{\perp}$, is also affected by nonlocality, and not only the in-plane component as predicted by KL. A discussion is given of some experimental results.

\section{The Kosztin-Leggett nonlocal effect}

The penetration depth would depend in some cases on the orientation of the sample with respect to the applied magnetic field. If the sample is aligned so that the $a b$ plane is perpendicular to the exciting field, screening currents are 
generated in the $a b$ plane and one measures the in-plane penetration depth $\lambda_{\|}(T)$. When the field is parallel to the $a b$ plane the measured signal is a combination of $\lambda_{\|}$and $\lambda_{\perp}$ : $\frac{\Delta \lambda(T)}{\lambda(0)}=\frac{\lambda_{\|}}{t}+\frac{\lambda_{\perp}}{w}$. Here $\lambda_{\perp}$, the out-of-plane penetration depth, corresponds to screening currents along the interlayer $c$ direction. The parameters $t$ and $w$ are the thickness and width of the sample, respectively.

To study the effect of nonlocal electrodynamics on the magnetic penetration depth, KL considered a weak, homogeneous magnetic field $\mathbf{H}$ applied along the $c$ axis of a semiinfinite HTSC with a plane boundary perpendicular to the $b$ axis (see Fig. 1). For a specular boundary

$$
\frac{\lambda(T)}{\lambda(0)}=\frac{2}{\pi} \int_{0}^{\infty} \frac{d q}{K(q, T)+q^{2}}
$$

where $K(q, T)$ is the response function to a weak, transverse vector potential. For a weak coupling, anisotropic superconductor [3]

$$
K(q, T)=2 \pi T \sum_{k}\left\langle\frac{k_{\|}^{2} \Delta_{k}^{2}}{\sqrt{w_{n}^{2}+\Delta_{k}^{2}}\left(w_{n}^{2}+\Delta_{k}^{2}+\gamma^{2}\right)}\right\rangle .
$$

Here, $w_{n}$ are the Matsubara frequencies, $\Delta_{k}$ is the gap function, $k_{\|}^{2}$ is the projection of $\mathbf{k}$ on the plane boundary, $\langle\ldots\rangle$ is an average over the Fermi surface, and $\gamma=\left(q \nu_{F} / 2\right) \mathbf{q} \cdot \mathbf{k}$. The parameter $\gamma$ determines the relevance of nonlocal electrodynamics: if $\gamma \neq 0(\gamma=0)$ nonlocality is (not) important. In the current configuration, screening currents are generated only in the planes, and being $\gamma \neq 0 \mathrm{KL}$ predicted that $\Delta \lambda_{\|}(T) \propto \Delta \lambda_{\| L}(T) T$ at temperatures $T<T_{N L}^{*}$. Here, $\Delta \lambda_{\| L}$ is the deviation of the in-plane penetration depth in the local approximation. Thus because $\Delta \lambda_{\| L} \propto T$ in $d$-wave superconductors, there should be in such superconductor a crossover from their characteristic linear $T$ behavior at low temperatures to a quadratic $T$ dependence at even lower temperatures. On the other hand, if the exciting field is oriented along the $b$ axis, as shown in Fig. 1, because of the semi-infinite space the only parameter would be again $\lambda_{\|}(T)$. However, in this case $\gamma=0$ and the in-plane penetration depth would not be affected by nonlocality. That is, for this orientation $\Delta \lambda_{\|}(T)=\Delta \lambda_{|| L}(T) \propto T$.

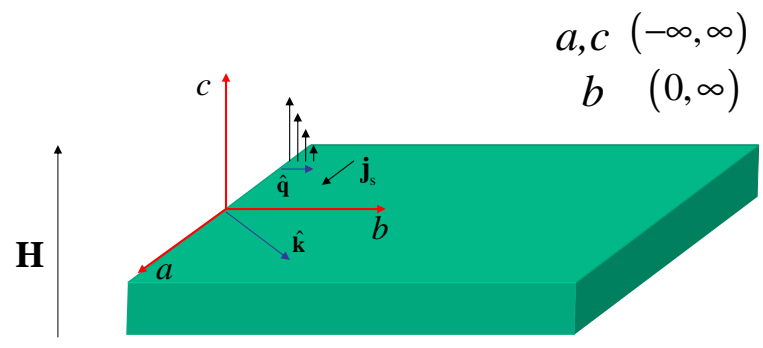

Figure 1. Magnetic field penetration and screening currents in a semi-infinite HTSC, as supposed by Kosztin and Leggett.
Consider now what happens in finite samples, which were not evaluated by KL. This is a quite important case, because the experimental samples are in fact small. If $\mathbf{H} \| c$, as shown in Fig. 2, the KL prediction for semi-infinite samples in this orientation holds. If $\mathbf{H} \perp c$, as indicated in Fig. 3, the prediction would be different from the one stated by $\mathrm{KL}$ for semi-infinite samples. In this orientation, $\Delta \lambda(T)$ is a combination of $\lambda_{\|}(T)$ and $\lambda_{\perp}(T)$, in the way mentioned above. The deviation of the in-plane penetration depth would follow $\Delta \lambda_{\|}(T)=\Delta \lambda_{\| L}(T) \propto T$, because $\gamma=0$ for screening currents along the plane. But $\gamma \neq 0$ for interlayer currents, so that $\lambda_{\perp}(T)$ should be modified by nonlocality. Thus, the statement that the effect of nonlocal electrodynamics is only relevant to $\lambda_{\|}(T)$ is no longer sustained in finite samples. Assuming that for an anisotropic $d$ wave superconductor (or any superconductor with nodes in the gap) the effect of nonlocal electrodynamics on the temperature dependence of $\lambda_{\perp}$ is similar to that of $\lambda_{\|}$, one has $\Delta \lambda_{\perp}(T) \propto \Delta \lambda_{\perp L}(T) T$ at temperatures $T<T_{N L}^{*}$. It must be pointed out that the $T_{N L}^{*}$ for the in- and out-of-plane components should be in general different. This indeed would be the case for anisotropic superconductors.

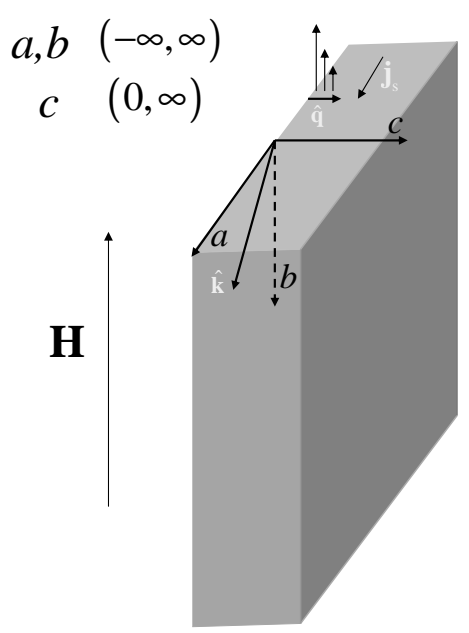

Figure 2. Magnetic field penetration and screening currents in a semi-infinite HTSC when $\mathbf{H} \perp c$.

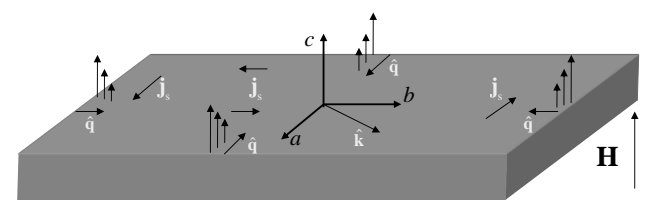

Figure 3. Magnetic field penetration and screening currents in a finite superconducting sample when $\mathbf{H} \| c$. 


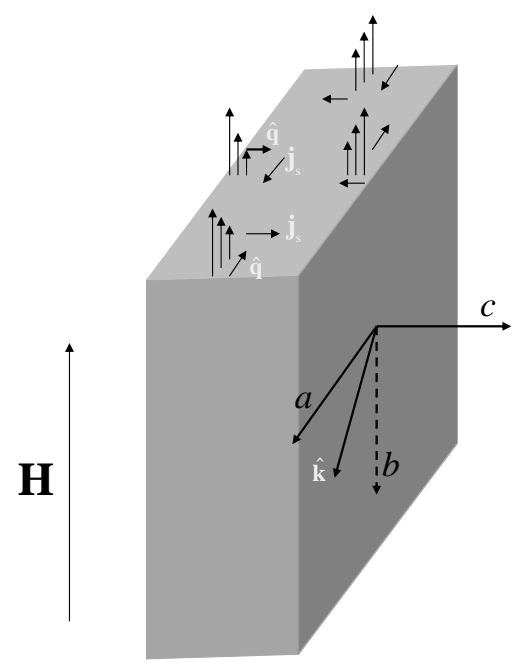

Figure 4. Magnetic field penetration and screening currents in a finite superconducting sample when $\mathbf{H} \perp c$.

\section{The $\mathrm{Sr}_{2} \mathrm{RuO}_{4}$ experimental data}

Although the KL effect was predicted with HTSC in mind, no studies of it using these superconductors have been reported. This is mainly due to limitations on the experimental resolution for $\lambda(T)$ in HTSC below $1 \mathrm{~K}\left(T_{\| N L}^{*} \sim 2 K\right.$ in YBCO for example).

The $\mathrm{Sr}_{2} \mathrm{RuO}_{4}$ penetration depth data [8] were the first showing some evidence for the KL nonlocal effect. These data, taken from samples with $T_{c}$ very close to the impurityfree $T_{c}=1.5 \mathrm{~K}$, can be reanalyzed by considering the contribution of the out-of-plane penetration depth when $\mathbf{H} \perp c$. It is worth to mention that $\mathrm{Sr}_{2} \mathrm{RuO}_{4}$ has nodes in the energy gap, although the exact symmetry of the gap is at the present time highly controversial. This material is a marginal local superconductor in the $a b$ plane, which leads to the expected $T_{\| N L}^{*} \simeq 1 \mathrm{~K}$. Measurements of $\Delta \lambda_{\|}(T)$ down to $30 \mathrm{mK}$ yielded a quadratic $\mathrm{T}$ dependence below $0.8 \mathrm{~K}$, as shown in Fig. 4. When the field was applied perpendicular to the $c$ axis, it was found $\Delta \lambda(T) \propto T^{2}$ below $0.8 \mathrm{~K}$ (see Fig. 5). Considering that $\lambda_{\perp} \simeq 20 \lambda_{\|}$, one could suppose that the signal was dominated by the out-of-plane penetration depth. That is, $\Delta \lambda(T) \sim \Delta \lambda_{\perp}(T) \propto T^{2}$. Assuming that the local $\lambda_{\perp}(T)$ is linear in $T$, this result would imply the presence of nonlocal effects. However, using the value $\lambda_{\perp}(0) / \xi_{0 \perp}=1123 T_{\perp N L}^{*}=2.4 \mathrm{mK}$, which is far below the observed $0.8 \mathrm{~K}$. This suggests that nonlocal effects on the out-of-plane $\lambda(T)$ in $\mathrm{Sr}_{2} \mathrm{RuO}_{4}$ should not be observed in the temperature range of the experiment $(T>30 \mathrm{mK})$. Thus, nonlocal effects may be discarded as a possible explanation for the $T^{2}$ dependence of the magnetic penetration depth of $\mathrm{Sr}_{2} \mathrm{RuO}_{4}$.

\section{Conclusions}

The effect of nonlocal electrodynamics on the magnetic penetration depth in superconductors with nodes in the gap has

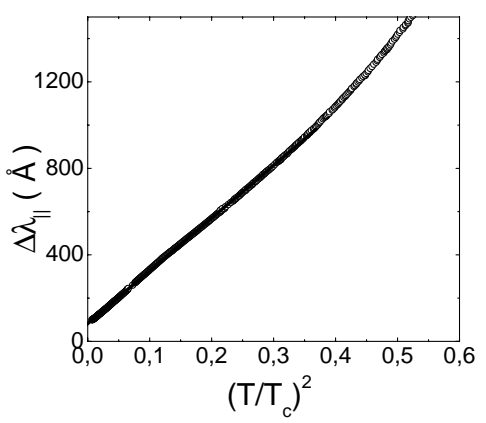

Figure 5. Low temperature dependence of the in-plane penetration depth in $\mathrm{Sr}_{2} \mathrm{RuO}_{4}$. (From Bonalde et al. [8]).

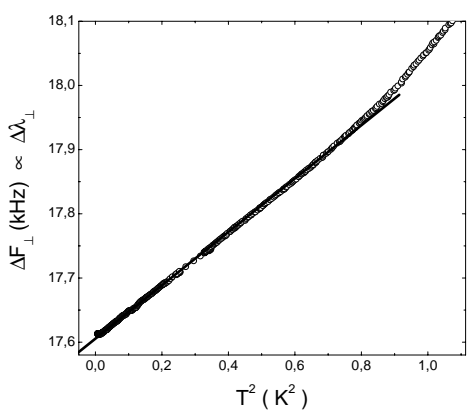

Figure 6. Low temperature dependence of the penetration depth in $\mathrm{Sr}_{2} \mathrm{RuO}_{4}$ when $\mathbf{H} \perp c$.

been revised for finite samples. It is found that not only the in-plane penetration depth $\lambda_{\|}(T)$ is affected by nonlocal electrodynamics, but also the out-of-plane penetration depth $\lambda_{\perp}(T)$. However, the temperature at which the nonlocal effect should be detected in $\lambda_{\perp}(T)$ could be much smaller than the one expected for $\lambda_{\|}(T)$.

\section{References}

[1] W. N. Hardy, D. A. Bonn, D. C. Morgan, R. Liang, and K. Zhang, Phys. Rev. Lett. 70, 3999 (1993).

[2] P. J. Hirschfeld and N. Goldenfeld, Phys. Rev. B 48, 4219 (1993).

[3] I. Kosztin and A. J. Leggett, Phys. Rev. Lett. 79, 135 (1997).

[4] F. Gross, B. S. Chandrasekhar, D. Eizel, K. Andres, P. J. Hirschfeld, H. R. Ott, J. Beuers, Z. Fisk, and J. L. Smith, Z. Phys. B 64, 175 (1986).

[5] D. A. Bonn, S. Kamal, K. Zhang, R. Liang, D. J. Baar, E. Klein, and W. N. Hardy, Phys. Rev. B 50, 4051 (1994).

[6] A. Carrington, I. Bonalde, R. Prozorov, R. W. Giannetta, A. M. Kini, J. Schlueter, H. H. Wang, U. Geiser, and J. M. Williams, Phys. Rev. Lett. 83, 4172 (1999).

[7] T. Xiang, C. Panagopoulos, and J. R. Cooper, Int. J. Mod. Phys. B 12, 1007 (1998).

[8] I. Bonalde, B. D. Yanoff, M. B. Salamon, D. J. V. Harlingen, E. E. M. Chia, Z. Q. Mao, and Y. Maeno, Phys. Rev. Lett. 85, 4775 (2000). 